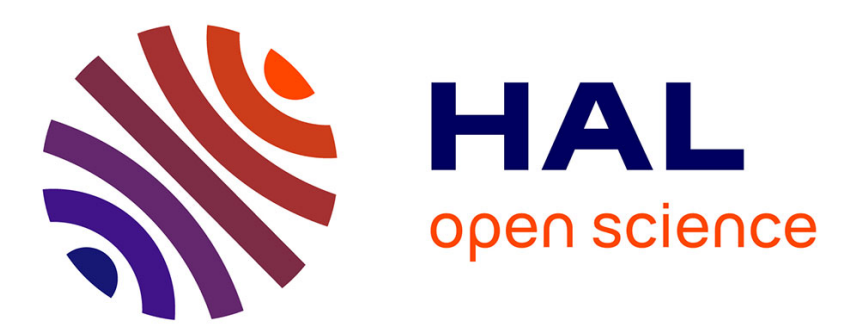

\title{
One-dimensional linear advection-diffusion equation: Analytical and finite element solutions
}

\author{
Abdelkader Mojtabi, Michel Deville
}

\section{To cite this version:}

Abdelkader Mojtabi, Michel Deville. One-dimensional linear advection-diffusion equation: Analytical and finite element solutions. Computers and Fluids, 2015, 107, pp.189-195. 10.1016/j.compfluid.2014.11.006 . hal-01331727

\section{HAL Id: hal-01331727 https://hal.science/hal-01331727}

Submitted on 14 Jun 2016

HAL is a multi-disciplinary open access archive for the deposit and dissemination of scientific research documents, whether they are published or not. The documents may come from teaching and research institutions in France or abroad, or from public or private research centers.
L'archive ouverte pluridisciplinaire HAL, est destinée au dépôt et à la diffusion de documents scientifiques de niveau recherche, publiés ou non, émanant des établissements d'enseignement et de recherche français ou étrangers, des laboratoires publics ou privés. 


\section{OATAO}

\section{Open Archive TOULOUSE Archive Ouverte (OATAO)}

OATAO is an open access repository that collects the work of Toulouse researchers and makes it freely available over the web where possible.

This is an author-deposited version published in : http://oatao.univ-toulouse.fr/ Eprints ID : 15920

To link to this article : DOI:10.1016/j.compfluid.2014.11.006

URL : http://dx.doi.org/10.1016/j.compfluid.2014.11.006

To cite this version : Mojtabi, Abdelkader and Deville, Michel Onedimensional linear advection-diffusion equation: Analytical and finite element solutions. (2015) Computers and Fluids, vol. 107. pp. 189-

195. ISSN 0045-7930

Any correspondence concerning this service should be sent to the repository administrator: staff-oatao@,listes-diff.inp-toulouse.fr 


\title{
One-dimensional linear advection-diffusion equation: Analytical and finite element solutions
}

\author{
Abdelkader Mojtabi ${ }^{\mathrm{a}, *}$, Michel O. Deville ${ }^{\mathrm{b}}$ \\ a Université Paul Sabatier and IMFT, 1 Avenue du Professeur Camille Soula, 31400 Toulouse, France \\ b École Polytechnique Fédérale de Lausanne, School of Engineering, Room MXD 130, Station 12, 1015 Lausanne, Switzerland
}

Keywords:

Separation of variables

Finite element method

Exponential layer

Symbolic computation

Asymptotic development

\begin{abstract}
A B S T R A C T
In this paper, a time dependent one-dimensional linear advection-diffusion equation with Dirichlet homogeneous boundary conditions and an initial sine function is solved analytically by separation of variables and numerically by the finite element method. It is observed that when the advection becomes dominant, the analytical solution becomes ill-behaved and harder to evaluate. Therefore another approach is designed where the solution is decomposed in a simple wave solution and a viscous perturbation. It is shown that an exponential layer builds up close to the downstream boundary. Discussion and comparison of both solutions are carried out extensively offering the numericist a new test model for the numerical integration of the Navier-Stokes equation.
\end{abstract}

\section{Introduction}

The numerical integration of the Navier-Stokes equations by standard methods like FXM (Finite X Methods), X being D(ifference), $\mathrm{E}$ (lement), $\mathrm{V}$ (olume) or by spectral and spectral elements requires a careful design. This is especially true and essential when long time integration ranges are involved as is the case for direct numerical simulation (DNS) or large-eddy simulations (LES) of turbulent flows where the evaluation of time averaged quantities imply very long time series to obtain meaningful information and statistics. Therefore temporal stability and space accuracy are the basic requirements needed to render the algorithms efficient on large scale parallel machines and to extract relevant physical phenomena.

The practitioners of computational fluid dynamics have decomposed the analysis of the complexity and stiffness of the NavierStokes equations into simpler problems like the Stokes (linear) equations that embody the difficulties of the space discretization of the velocity and pressure fields and the advection-diffusion problem that is related to the transport character of the non-linear terms. This last class of problems includes the non-linear Burgers equations and the linear advection-diffusion (LAD) equation. In this paper, we will address the one-dimensional LAD equation with

* Corresponding author.

E-mail addresses: mojtabi@imft.fr (A. Mojtabi), michel.deville@epfl.ch (M.O. Deville). homogeneous Dirichlet boundary conditions as this is a meaningful test for established or novel discrete schemes. For high Reynolds number flows the advection is dominating diffusion but the presence of the boundaries imposing no-slip wall conditions complicates the solution of the problem. Boundary layers develop and in most cases influence deeply the flow dynamics. No-slip wall boundary conditions impede the general use of periodic Fourier representation and spectral calculation.

Even though the LAD equation is linear it is difficult to find closed form analytical solution in the literature. Most of the efforts have been devoted to the solution of LAD with an upstream boundary condition and a Robin or Neumann downstream condition. The presence of the gradient condition at the exit of the domain eases the development of the analytical solution. The paper by Pérez Guérrero et al. [10] uses a change of variable to obtain a heat equation which is then solved by a generalized integral transform technique proposed by Cotta [4]. In [13], van Genuchten et al. are able to use a variable transformation that reduces the partial differential equation to an ordinary differential equation the solution of which is expressed by the complementary error function. Other methodologies are possible to tackle the LAD problem on finite or infinite domains. Without the pretension of being exhaustive, we can cite Bosen [3], Kumar et al. [8], Pérez Guérrero et al. [11] and Zoppou and Knight [14].

On the numerical side, finite differences have been applied, see for example [5]. In the finite element framework, Gresho et al. [7] investigate a time integrator based on the combination of the 
trapezoid rule and the second-order Adams-Bashforth scheme with piecewise linear elements for space discretization. Some analytical solutions are presented in the various examples solved throughout the paper. However none of them treats the LAD problem with homogeneous Dirichlet conditions and a smooth initial condition like a sine function. In the book of Donea and Huerta [6] the LAD problem is proposed with a truncated Gaussian profile as the initial condition.

In this paper we will solve the LAD problem with homogeneous boundary conditions and a sine profile for the initial condition. This is exactly the same initial and boundary conditions that were imposed for the Burgers equation solved by Basdevant et al. [2]. We will be able to compare the physics associated with both problems. The paper is organized as follows. Section 2 describes the LAD problem which is solved in closed form by the introduction of a change of variables. Section 3 details the analytical solution when the viscosity goes to zero. In this case the problem at hand is a simple wave equation perturbed by the presence of a very weak viscous term. Section 4 presents the Fourier solution when periodic conditions are applied. Section 5 is devoted to some considerations related to energy conservation. Section 6 treats the numerical method obtained by linear finite elements and a time integration using a Crank-Nicolson scheme for the viscous term and a second order Adams-Bashforth scheme for the advection term. Section 7 reports the results produced by both approaches and compares them. Finally the last section draws conclusions.

\section{Linear advection-diffusion equation}

The unsteady linear advection-diffusion equation is given by the following relation

$\left.\left.\frac{\partial u}{\partial t}+c \frac{\partial u}{\partial x}=v \frac{\partial^{2} u}{\partial x^{2}}, \quad-1<x<1, \quad t \in\right] 0, T\right]$,

where $u$ is the velocity variable, $c>0$ the constant advection velocity, $v$ the kinematic viscosity and time $t$. We will impose homogeneous Dirichlet boundary conditions $u(-1, t)=u(1, t)=0$ and the initial condition $u(x, 0)=-\sin \pi x$. These initial and boundary conditions were already used for the Burgers equation in [2]. This choice will allow us to compare the two cases.

To obtain a closed form solution, let us make the change of variables

$u(x, t)=v(x, t) e^{\alpha x+\beta t}$.

Introducing (2) in (1) and simplifying by the exponential, one obtains

$\frac{\partial v}{\partial t}+\left(\beta+c \alpha-\alpha^{2} v\right) v+(c-2 \alpha v) \frac{\partial v}{\partial x}=v \frac{\partial^{2} v}{\partial x^{2}}$.

As $\alpha$ and $\beta$ are free parameters, we choose them in such a way that

$\beta+c \alpha-\alpha^{2} v=0$

$c-2 \alpha \nu=0$.

Therefore, $\alpha=c / 2 v$ and $\beta=-c^{2} / 4 v$. The governing equation for $v$ is reduced to the standard heat equation

$\frac{\partial v}{\partial t}=v \frac{\partial^{2} v}{\partial x^{2}}$

subject to the homogeneous conditions $v(-1, t)=v(1, t)=0$ and the initial condition

$v(x, 0)=-\sin \pi x e^{-\alpha x}=-\sin \pi x e^{-\frac{c x}{2 v}}$.

Let us use the method of separation of variables to solve (6) by setting $v(x, t)=X(x) T(t)$. Omitting the details of the algebra, this leads to the solution $v(x, t)=\sum_{k=0}^{\infty}\left(A_{k} \sin \frac{k \pi x}{2}+B_{k} \cos \frac{k \pi x}{2}\right) e^{-v \frac{k^{2} \pi^{2}}{4} t}$.

The boundary conditions impose the conditions $A_{2 p+1}=B_{2 p}=0, p=0,1, \ldots$ Eq. (8) becomes

$v(x, t)=\sum_{p=0}^{\infty} A_{2 p} \sin (p \pi x) e^{-v p^{2} \pi^{2} t}+B_{2 p+1} \cos \left(\frac{2 p+1}{2} \pi x\right) e^{-v \frac{(2 p+1)^{2}}{4} \pi^{2} t}$.

Applying the initial condition (7) to Eq. (9) yields

$\sum_{p=0}^{\infty} A_{2 p} \sin p \pi x+B_{2 p+1} \cos \frac{2 p+1}{2} \pi x=-\sin (\pi x) e^{-\frac{c x}{2 v}}$.

Using the orthogonality property of Fourier polynomials, the coefficients $A_{2 p}$ and $B_{2 p+1}$ are obtained solving the relations

$A_{2 p} \int_{-1}^{1}(\sin p \pi x)^{2} d x=-\int_{-1}^{1} \sin (\pi x) \sin (p \pi x) e^{-\frac{c x}{2 v}} d x$

$B_{2 p+1} \int_{-1}^{1}\left(\cos \frac{2 p+1}{2} \pi x\right)^{2} d x=-\int_{-1}^{1} \sin (\pi x) \cos \left(\frac{2 p+1}{2} \pi x\right) e^{-\frac{c x}{2 v}} d x$

With the help of standard trigonometric relations, the right hand side integral of (11) may be rewritten as

$\int_{-1}^{1} \sin \pi x \sin p \pi x e^{-\frac{c x}{2 v}} d x=\frac{1}{2} \int_{-1}^{1}[\cos (p-1) \pi x-\cos (p+1) \pi x] e^{-\frac{c x}{2 v}} d x$.

Furthermore one has also the identity (cf. [1])

$\int e^{-a x} \cos p \pi x d x=\frac{e^{-a x}}{a^{2}+p^{2} \pi^{2}}(-a \cos p \pi x+p \pi \sin p \pi x)$.

Therefore one gets

$A_{2 p}=\frac{-32(-1)^{p+1} v^{3} c \pi^{2} p \sinh (c / 2 v)}{c^{4}+8(c \pi v)^{2}\left(p^{2}+1\right)+16(\pi v)^{4}\left(p^{2}-1\right)^{2}}$.

A similar development gives

$B_{2 p+1}=\frac{-16(-1)^{p+1} v^{3} c \pi^{2}(2 p+1) \cosh (c / 2 v)}{c^{4}+(c \pi v)^{2}\left(8 p^{2}+8 p+10\right)+(\pi v)^{4}\left(4 p^{2}+4 p-3\right)^{2}}$.

With (2) and the relations (9), (15), (16) one writes

$u(x, t)=16 \pi^{2} v^{3} c e^{\frac{c}{2 v}\left(x-\frac{c}{2} t\right)}$

$$
\begin{aligned}
& \times\left[\sinh \left(\frac{c}{2 v}\right) \sum_{p=0}^{\infty} \frac{(-1)^{p} 2 p \sin (p \pi x) e^{-v p^{2} \pi^{2} t}}{c^{4}+8(c \pi v)^{2}\left(p^{2}+1\right)+16(\pi v)^{4}\left(p^{2}-1\right)^{2}}\right. \\
& \left.+\cosh \left(\frac{c}{2 v}\right) \sum_{p=0}^{\infty} \frac{(-1)^{p}(2 p+1) \cos \left(\frac{2 p+1}{2} \pi x\right) e^{-v \frac{(2 p+1)^{2}}{4} \pi^{2} t}}{c^{4}+(c \pi v)^{2}\left(8 p^{2}+8 p+10\right)+(\pi v)^{4}\left(4 p^{2}+4 p-3\right)^{2}}\right] .
\end{aligned}
$$

When the viscosity goes to zero, the solution becomes

$$
\begin{aligned}
& u(x, t)=8 \pi^{2}\left(\frac{v}{c}\right)^{3} e^{\frac{c}{2 v}\left((x+1)-\frac{c}{2} t\right)} \\
& {\left[\sum_{p=0}^{\infty}(-1)^{p}\left(2 p \sin (p \pi x)+(2 p+1) \cos \left(\frac{2 p+1}{2} \pi x\right)\right)\right] .}
\end{aligned}
$$

We observe that the presence of the exponential term in (18) renders the problem stiffer and the closed form solution blows up for vanishing viscosity. This ill-behavior requires a special treatment.

\section{Analytical solution for vanishing viscosity}

We will decompose the problem solution in two parts

$u(x, t)=u_{a}(x, t)+v U(x, t)$ 
where $u_{a}$ indicates the solution of the advection equation, i.e. when $v=0$, and $U$ a viscous contribution. Obviously the solution for $u_{a}$ is given as

$u_{a}= \begin{cases}-\sin (\pi(x-c t)) & \text { for } c t<x+1 \\ 0 & \text { for } \quad c t \geqslant x+1 .\end{cases}$

Insertion of (19) in (1) yields

$\frac{\partial u_{a}}{\partial t}+c \frac{\partial u_{a}}{\partial x}+v\left(\frac{\partial U}{\partial t}+c \frac{\partial U}{\partial x}\right)=v \frac{\partial^{2} u_{a}}{\partial x^{2}}+v^{2} \frac{\partial^{2} U}{\partial x^{2}}$.

The first two terms vanish as they satisfy the wave equation. Neglecting the second order term in $v^{2}$, we are left with

$\frac{\partial U}{\partial t}+c \frac{\partial U}{\partial x}=\frac{\partial^{2} u_{a}}{\partial x^{2}}$.

Taking (20) into account, Eq. (22) reads

$\frac{\partial U}{\partial t}+c \frac{\partial U}{\partial x}=\delta \pi^{2} \sin (\pi(x-c t))$ with $\begin{cases}\delta=1 & \text { for } \quad c t<x+1 \\ \delta=0 & \text { for } \quad c t \geqslant x+1 .\end{cases}$

For this first-order equation, it is easy to verify that the initial and boundary conditions for $U$ are

$$
\begin{aligned}
U(x, 0) & =0, \quad-1<x<1 \\
U(-1, t) & =0, \quad t \geqslant 0 .
\end{aligned}
$$

We can also verify that on the right boundary, the solution must reach the following value

$U(1, t)= \begin{cases}\frac{1}{v} \sin (\pi c t) & \text { for } c t<2 \\ 0 & \text { for } c t \geqslant 2 .\end{cases}$

\subsection{Case $\delta=0,(c t \geqslant x+1)$}

\subsection{1. $c t \geqslant 2$}

With (23)-(25), we obtain the solution

$U(x, t)=0, \quad-1<x<1$.

\subsection{2. $c t<2$}

Referring to the same relations, the solution is

$U(x, t)=0, \quad$ for $\quad-1 \leqslant x+1, \quad$ and $\quad U(1, t)=\frac{1}{v} \sin (\pi c t)$.

3.2. Case $\delta=1,(c t<x+1)$

To solve

$\frac{\partial U}{\partial t}+c \frac{\partial U}{\partial x}=\pi^{2} \sin (\pi(x-c t))$,

with (24) and (25), we set

$U(x, t)=\pi^{2} c t \sin (\pi(x-c t))+V(x, t)$,

where the first term in the right hand side is a particular solution. This procedure gives the equation

$\frac{\partial V}{\partial t}+c \frac{\partial V}{\partial x}=0$

with the conditions

$V(x, 0)=0, \quad-1<x<1$

$V(-1, t)=-\pi^{2} c t \sin (\pi c t)$.

The $V$ solution is

$V(x, t)=-\pi^{2}(c t-x-1) \sin (\pi(x-c t))$ for $\quad c t<x+1$.
In this case, the final expression for $U(x, t)$ is

$U(x, t)=\pi^{2}(x+1) \sin (\pi(x-c t))$.

Consequently the LAD solution for very small viscosity becomes

$u(x, t)= \begin{cases}-\sin (\pi(x-c t))\left(1-v \pi^{2}(x+1)\right) & \text { for } c t<x+1 \\ 0 & \text { for } c t \geqslant x+1\end{cases}$

\section{Fourier solution}

Let us assume that instead of Dirichlet conditions, the problem is subject to periodic conditions. Then the Fourier solution is readily obtained

$u(x, t)=-\sin (\pi(x-c t)) e^{-v \pi^{2} t}$.

If the viscosity goes to zero, the periodic solution becomes

$u(x, t)=-\sin (\pi(x-c t))\left(1-v \pi^{2} t+O\left(v^{2}\right)\right)$,

a relation that has some similarities with Eq. (36).

\section{The energy equation}

Here we look at the conservation law for the kinetic energy of the problem. Multiplying Eq. (1) through by $u$ and integrating over the domain, one gets

$$
\begin{aligned}
\int_{-1}^{1}\left(\frac{\partial}{\partial t}\left(\frac{u^{2}}{2}\right)+c \frac{\partial}{\partial x}\left(\frac{u^{2}}{2}\right)\right) d x & =v \int_{-1}^{1} u \frac{\partial^{2} u}{\partial x^{2}} \\
& =-v \int_{-1}^{1}\left(\frac{\partial u}{\partial x}\right)^{2} d x
\end{aligned}
$$

where integration by parts was used to evaluate the last term. Carrying through the integration, the energy equation reduces to

$\frac{d}{d t} \int_{-1}^{1} \frac{u^{2}}{2} d x=-v \int_{-1}^{1}\left(\frac{\partial u}{\partial x}\right)^{2} d x$

As is well known the energy decay is governed by the diffusion.

It is also possible to compute the time needed to reach a zero velocity at each point of the domain. For this purpose, we integrate (1)

$\int_{-1}^{1}\left(\frac{\partial u}{\partial t}+c \frac{\partial u}{\partial x}\right) d x=v \int_{-1}^{1} \frac{\partial^{2} u}{\partial x^{2}} d x$

leading to the relation

$\frac{d}{d t} \int_{-1}^{1} u d x:=\frac{d \underline{U}}{d t}=v\left[\frac{\partial u}{\partial x}(1, t)-\frac{\partial u}{\partial x}(-1, t)\right]$,

where $\underline{U}$ denotes the average velocity. Consequently we obtain

$\underline{U}(t)=v \int_{0}^{t}\left[\frac{\partial u}{\partial x}(1, t)-\frac{\partial u}{\partial x}(-1, t)\right] d t$.

We deduce the value $T_{f}$ which corresponds to $\underline{U}\left(t=T_{f}\right)=0$.

\section{Finite element method}

The finite element (FE) method is based on the weak formulation of the problem (1). Choosing $v$ as the test function, we write

$\int_{-1}^{1}\left[\left(\frac{\partial u}{\partial t}+c \frac{\partial u}{\partial x}\right) v+v \frac{\partial u}{\partial x} \frac{\partial v}{\partial x}\right] d x=0$.

The global FE approximation is sought in terms of linear Lagrange polynomials making up hat functions such that the numerical discretization is 
$u_{h}(x)=\sum_{i=1}^{E+1} u_{i}(t) \varphi_{i}(x), \quad-1 \leqslant x \leqslant 1$,

where $h$ refers to the FE grid size. The subscript $i$ denotes the index of the FE grid nodes and $E$ is the number of elements covering the computational domain $-1 \leqslant x \leqslant 1$. The variables $u_{i}(t)$ are the time dependent nodal values. The hat functions are linear interpolants such that

$\varphi_{i}(x)=\left\{\begin{array}{ll}\frac{x-x_{i-1}}{h} & x \in\left[x_{i-1}, x_{i}\right] \\ \frac{x_{i+1}-x}{h} & x \in\left[x_{i}, x_{i+1}\right] \\ 0 & x \notin\left[x_{i-1}, x_{i+1}\right]\end{array}, \quad 2 \leqslant i \leqslant E\right.$.

The number of grid points is therefore $N=E+1$. In the framework of the Galerkin method, the test functions are chosen the same as the approximation polynomials. The discrete problem becomes

$\int_{-1}^{1}\left[\left(\frac{\partial u_{h}}{\partial t}+c \frac{\partial u_{h}}{\partial x}\right) v_{h}+v \frac{\partial u_{h}}{\partial x} \frac{\partial v_{h}}{\partial x}\right] d x=0$,

with $v_{h}=\varphi_{i} i=1, \ldots, E$. Introducing (46) in (47), we generate a linear system of algebraic equations of order $N-2$ as the end points are given boundary values

$\sum_{k=2}^{N-1}\left(M_{i, k} \frac{d u_{k}}{d t}+\left(c D_{i, k}+v K_{i, k}\right) u_{k}\right)=0, \quad 2 \leqslant i \leqslant N-1$.

It is a simple exercise to compute the various matrices involved in the discrete equations. The stiffness matrix $[K]$ is symmetric and positive definite

$K_{i, k}=\int_{-1}^{1} \frac{d \varphi_{i}}{d x} \frac{d \varphi_{k}}{d x} d x, \quad 2 \leqslant i, k \leqslant N-1$.

The mass matrix $[M]$ is given as

$M_{i, k}=\int_{-1}^{1} \varphi_{i} \varphi_{k} d x, \quad 2 \leqslant i \leqslant N-1$,

while the weak derivative $[D]$ corresponds to

$D_{i, k}=\int_{-1}^{1} \frac{d \varphi_{i}}{d x} \varphi_{k} d x, \quad 2 \leqslant i \leqslant N-1$.

The discrete equation for node $i$ is

$\frac{h}{6} \frac{d u_{i-1}}{d t}+\frac{2 h}{3} \frac{d u_{i}}{d t}+\frac{h}{6} \frac{d u_{i+1}}{d t}+c \frac{u_{i+1}-u_{i-1}}{2}-\frac{v}{h}\left(u_{i-1}-2 u_{i}+u_{i+1}\right)=0$.

The resulting set of ordinary differential equations reads

$[M] \frac{d \underline{u}(t)}{d t}+([K]+[D]) \underline{u}(t)=0$,

where $\underline{u}$ is the vector collecting all problem unknowns. Using an implicit Crank-Nicolson time scheme for the viscous part and a second-order Adams-Bashforth explicit integration for the advection term, the full discrete equations read

$\left([M]+\frac{\Delta t}{2}[K]\right) \underline{u}^{n+1}=\left([M]-\frac{\Delta t}{2}[K]\right) \underline{u}^{n}+\frac{\Delta t}{2}[D]\left(3 \underline{u}^{n}-\underline{u}^{n-1}\right)$,

with the time step $\Delta t$ submitted to a CFL condition for stability considerations of the explicit part.

\section{Results}

We will set up $c=1$ for the sake of simplicity. This choice has no consequences on the generality of the discussion. The Reynolds number is defined as

$R e=\frac{c L}{v}=\frac{2}{v}$,

with the choice $L=2$.
The analytical solution (17) is computed by Maple ${ }^{\odot}$ [9] with 800 terms in the series. For the moderate values of $v=1 / 10 \pi, 1 / 100$ the computations are carried out with 50 digits to reach the desired accuracy, namely 16 significant decimal places. For the case $v=1 / 100 \pi, 150$ digits are necessary, while 500 digits are needed for $v=1 / 1000$. If 400 digits were used in this last computation, the analytical solution blows up due to the instability and the stiffness induced by the presence of the exponential $e^{\frac{c}{2 v} x}$.

The FE solution is obtained with $E=1000$ elements and $\Delta t=1 / 10$ for $v=1 / 10 \pi$ and $\Delta t=1 / 100$ for $v=1 / 100 \pi$.

Figs. 1 and 2 show the analytical solution in colored solid lines for $v=1 / 10 \pi$ and $v=1 / 100$, respectively. The black dots show the FE solution for equally spaced abscissae with $\Delta x=0.05$. The agreement between both solutions is good and the maximum error in $L^{\infty}$ norm is of the order of $10^{-2}$. Fig. 3 shows the solutions for $v=1 / 100 \pi$. Note that close to $x=1$ a very sharp boundary layer develops. The LAD solution is very different from the one computed for the non-linear Burgers equation where $c=u$. In this case, the maxima of the sine function move in the direction of the origin and build up a sawtooth profile which is still $C^{\infty}$ by the presence of the viscosity. The sawtooth becomes discontinuous only for the inviscid case.

The FE solution for $v=1 / 1000$ requires $E=2000$ elements and $\Delta t=1 / 100$. Fig. 4 illustrates the comparison of the analytical and FE solutions. We conclude that the boundary layer at $x=1$ behaves like a sharp exponential layer.

Let us now inspect the solution (36) for weak viscosity.

Fig. 5 compares the $u$ profile for the three values of $v=0,1 / 100 \pi$ and $v \rightarrow 0$. The inviscid solution has no damping as it corresponds to a simple wave. The solution for the vanishing viscosity has a sharp corner at $x=0$ which is completely smeared by the viscosity for $v=1 / 100 \pi$.

The rate of change of the kinetic energy is governed by viscosity and the gradient $\partial u / \partial x$, cf. Eq. (40). Of major importance is the slope of the profile at $x=1$. From Eq. (17), the exponential layer thickness goes like $O\left(e^{\frac{c}{2 v}}\right)$. Figs. 6 and 7 display the variation of the slope versus time. Starting from the initial value $\pi$, for $v=1 / 100 \pi$, the maximum value reaches 309.402 at $t=0.49045$, while the minimum value -299.8333 is obtained at $t=1.4904$ The FE values with $E=1000$ elements, computed by a backward second order finite difference formula, yield 291.72 at $t=0.49$ and -282.698 at $t=1.49$. With $E=2000$ elements, one has 303.73 and -294.31 at the same times.

For $v=1 / 1000$, the maximum value reaches 994.914 at $t=0.4905$, while the minimum value -985.143 is obtained at $t=1.4905$.

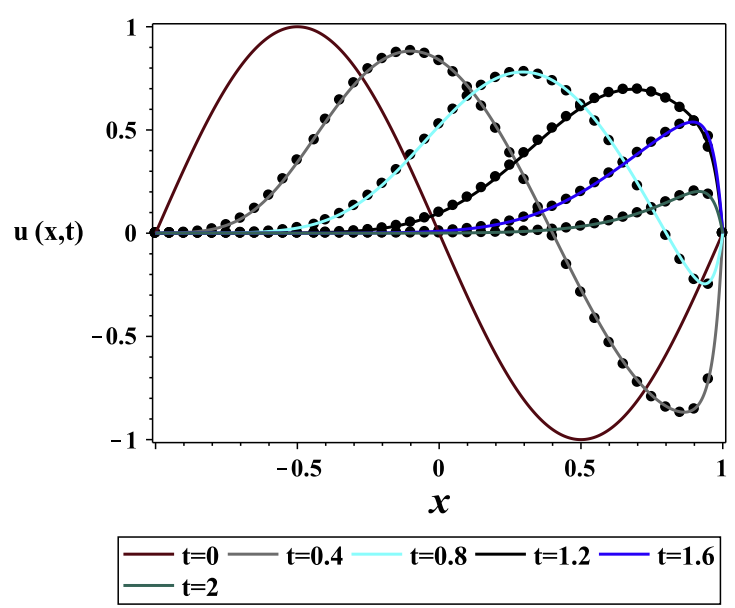

Fig. 1. Analytical and numerical solutions for $R e=20 \pi$. 


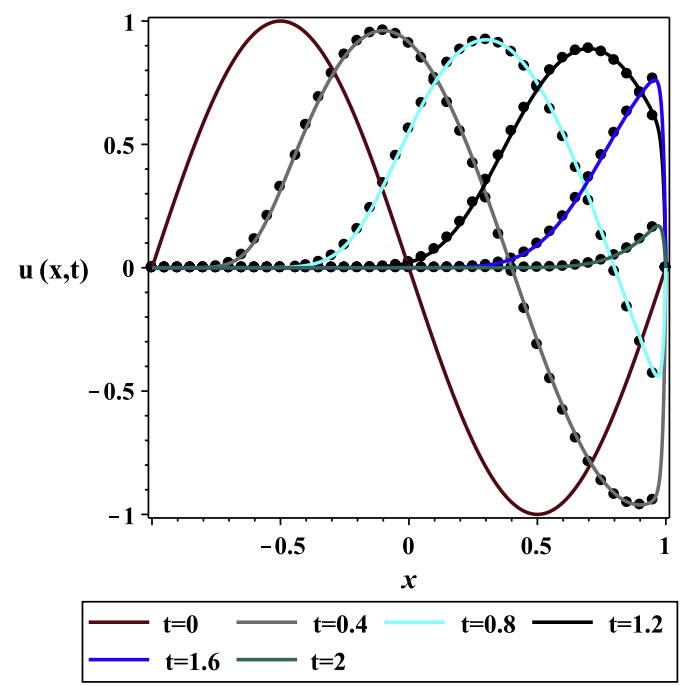

Fig. 2. Analytical and numerical solutions for $R e=200$.

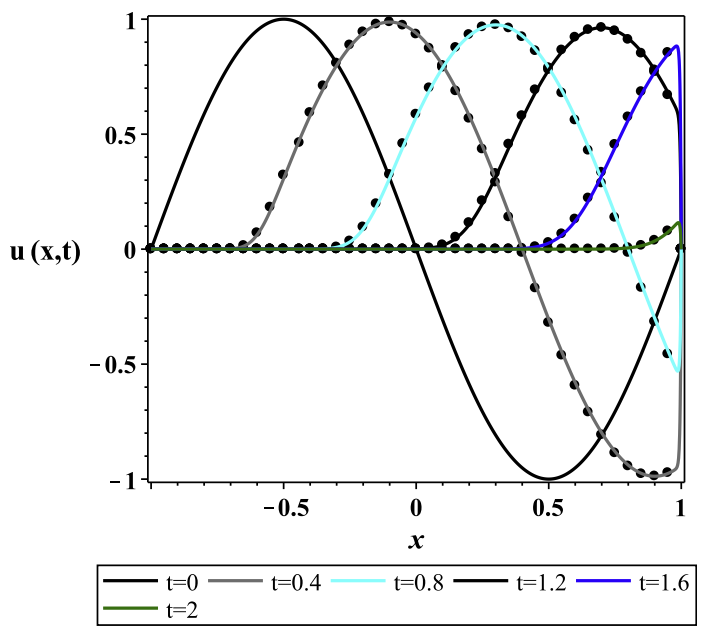

Fig. 3. Analytical and numerical solutions for $R e=200 \pi$.

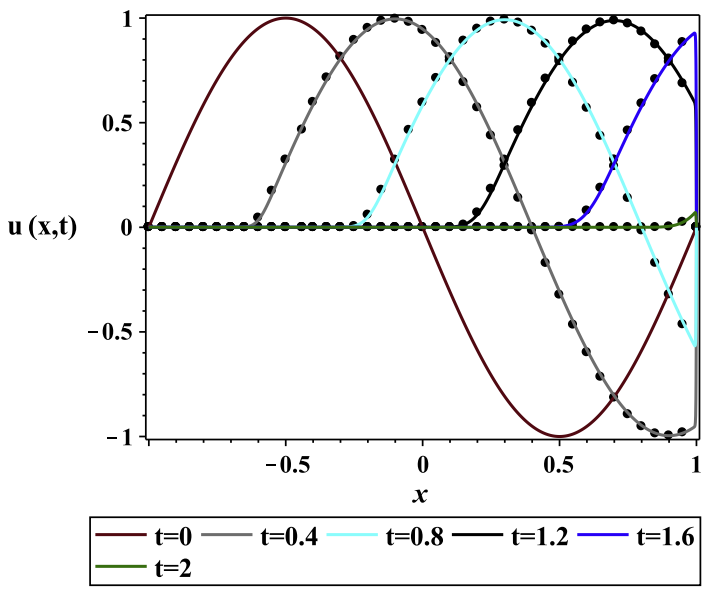

Fig. 4. Analytical and numerical solutions for $R e=2000$.

Fig. 8 exhibits the time variation of the average velocity. The final time $T_{f}$ for the values $v=1 / 10 \pi, 1 / 100,1 / 100 \pi$, respectively are given by $T_{f}=2.787,2.423,2.204$.

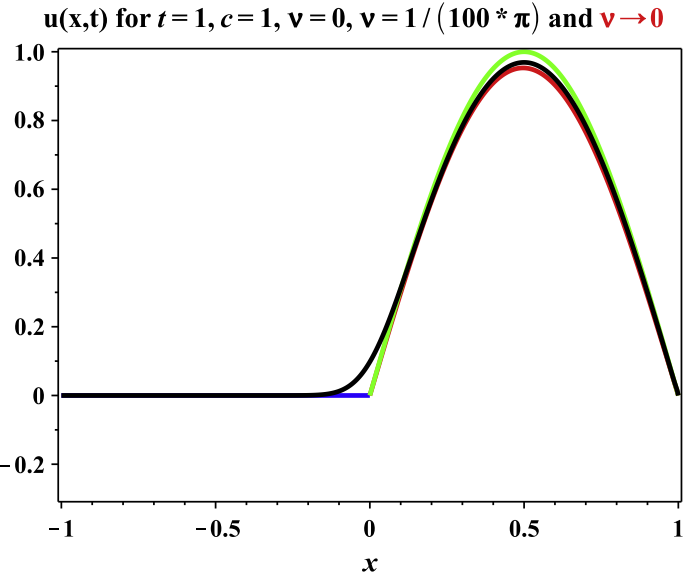

Fig. 5. Analytical solutions for $t=1, v=0$ (green), $v=1 / 100 \pi$ (black), $v \rightarrow 0$ (red). (For interpretation of the references to colour in this figure legend, the reader is referred to the web version of this article.)

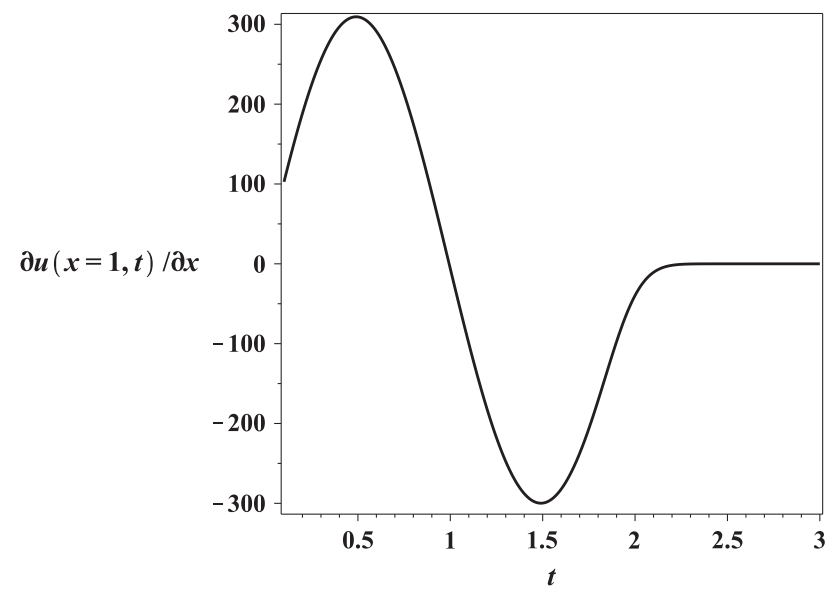

Fig. 6. $\frac{\partial u}{\partial x}(x=1, t), v=1 / 100 \pi$.

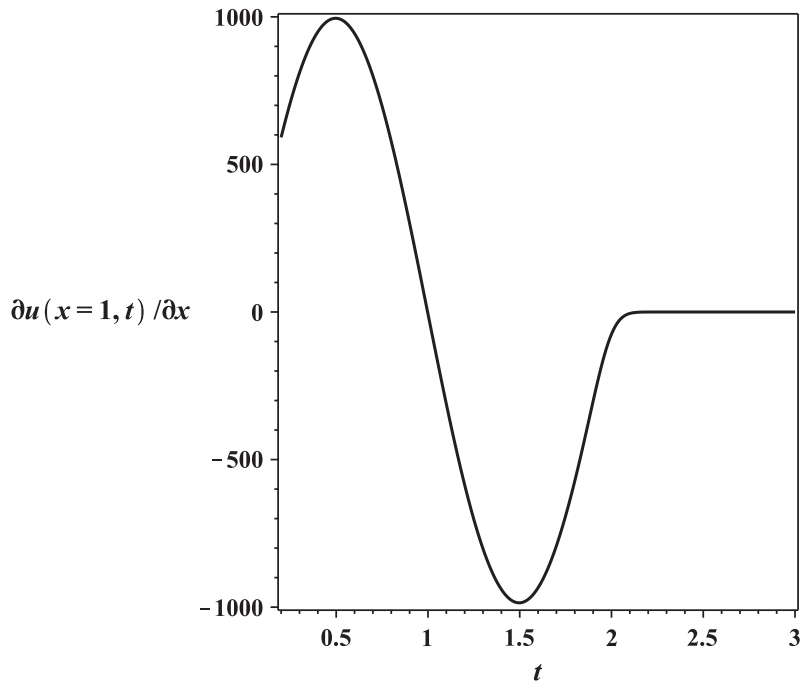

Fig. 7. $\frac{\partial u}{\partial x}(x=1, t), v=1 / 1000$.

Tables 1-3 give numerical values with five accurate decimal places of the $u$ solution (17) at times $t=0.8,1.0,1.6$ and Reynolds numbers $40 \pi, 400,200 \pi, 4000$. One observes that the solu- 


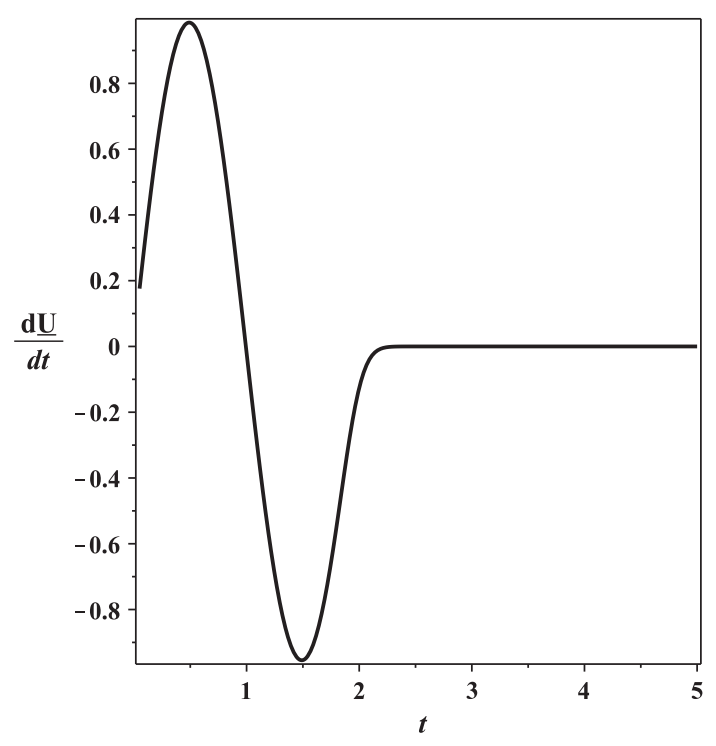

Fig. 8. $\frac{d \underline{U}}{d t}$ versus time, $v=1 / 100 \pi$.

Table 1

Analytical results for $u(0.9 \leqslant x<1, t=0.8), \operatorname{Re}=40 \pi, 400,200 \pi, 4000$.

\begin{tabular}{lllll}
\hline$x$ & $R e=40 \pi$ & $R e=400$ & $R e=200 \pi$ & $R e=4000$ \\
\hline 0.9 & -0.27119 & -0.29706 & -0.30516 & -0.30780 \\
0.94 & -0.36068 & -0.40929 & -0.42046 & -0.42410 \\
0.96 & -0.37596 & -0.46288 & -0.47574 & -0.47986 \\
0.98 & -0.31256 & -0.50386 & -0.52913 & -0.53372 \\
0.99 & -0.20734 & -0.46059 & -0.55393 & -0.55987 \\
0.999 & -0.00445 & -0.09798 & -0.26693 & -0.50336 \\
\hline
\end{tabular}

Table 2

Analytical results for $u(0.4 \leqslant x<1, t=1.0), R e=40 \pi, 400,200 \pi, 4000$.

\begin{tabular}{lllll}
\hline$x$ & $R e=40 \pi$ & $R e=400$ & $R e=200 \pi$ & $R e=4000$ \\
\hline 0.4 & 0.81507 & 0.90527 & 0.93623 & 0.94637 \\
0.5 & 0.85503 & 0.95185 & 0.98441 & 0.99508 \\
0.6 & 0.81286 & 0.90526 & 0.93623 & 0.94637 \\
0.7 & 0.69142 & 0.77006 & 0.79641 & 0.80503 \\
0.8 & 0.5023 & 0.55948 & 0.57862 & 0.58489 \\
0.9 & 0.26459 & 0.29414 & 0.30420 & 0.30750 \\
0.94 & 0.16383 & 0.17836 & 0.18446 & 0.18646 \\
0.98 & 0.06894 & 0.06086 & 0.06181 & 0.06248 \\
0.99 & 0.04117 & 0.03394 & 0.03098 & 0.03126 \\
0.999 & 0.00521 & 0.00544 & 0.00474 & 0.00355 \\
\hline
\end{tabular}

Table 3

Analytical results for $u(0.9 \leqslant x<1, t=1.6), R e=40 \pi, 400,200 \pi, 4000$

\begin{tabular}{lllll}
\hline $\mathrm{x}$ & $R e=40 \pi$ & $R e=400$ & $R e=200 \pi$ & $R e=4000$ \\
\hline 0.90 & 0.62300 & 0.74874 & 0.78894 & 0.80265 \\
0.94 & 0.68241 & 0.81004 & 0.85456 & 0.86941 \\
0.96 & 0.65665 & 0.83652 & 0.88237 & 0.89771 \\
0.98 & 0.51887 & 0.81925 & 0.90670 & 0.92246 \\
0.99 & 0.33970 & 0.68929 & 0.91578 & 0.93348 \\
0.999 & 0.04440 & 0.22293 & 0.43121 & 0.81478 \\
\hline
\end{tabular}

tion for $t=0.8$ and 1.6 and $R e=4000$ builds up an extremely sharp variation near the right boundary. In Table 4 we report the slope of the $u$ profile at $x=1$ for the times $t=0.8,1.0$ and 1.6.

Let us inspect the accuracy achieved by the FE solution for the slope $\frac{\partial u}{\partial x}(1, t)$. We will examine two intermediate values of Re given
Table 4

Analytical results for $\frac{\partial u}{\partial x}(1, t), \operatorname{Re}=40 \pi, 400,200 \pi, 2000,4000$.

\begin{tabular}{rrrrrr}
\hline$\frac{\partial u}{\partial x}(1, t)$ & $R e=40 \pi$ & $R e=400$ & $R e=200 \pi$ & $R e=2000$ & $R e=4000$ \\
\hline$t=0.8$ & 28.087 & 108.120 & 175.118 & 578.119 & 1165.876 \\
$t=1.0$ & -5.370 & -5.981 & -6.089 & -6.221 & -6.252 \\
$t=1.6$ & -45.814 & -173.998 & -282.290 & -934.245 & -1885.227 \\
\hline
\end{tabular}

Table 5

FE results for $\frac{\partial u}{\partial x}(1, t), R e=200 \pi$.

\begin{tabular}{llll}
\hline$E$ & $t=0.8$ & 1.0 & 1.6 \\
\hline 2000 & 171.9858 & -5.8412 & -277.1557 \\
4000 & 174.2134 & -6.0202 & -280.8124 \\
\hline
\end{tabular}

Table 6

FE results for $\frac{\partial u}{\partial x}(1, t), R e=2000$.

\begin{tabular}{llll}
\hline$E$ & $t=0.8$ & 1.0 & 1.6 \\
\hline 4000 & 555.1425 & -5.7787 & -896.9483 \\
8000 & 571.0469 & -6.0448 & -922.7606 \\
\hline
\end{tabular}

in Table 4, namely $R e=200 \pi$ and $R e=2000$. Table 5 presents the numerical values for $R e=200 \pi$ with two discretizations $E=2000$ and $E=4000$. Richardson extrapolation of $\frac{\partial u}{\partial x}(1, t=1.6)$ yields the value -282.0313 , offering three significant digits. Table 6 shows the results for $R e=2000$ with $E=4000$ and $E=8000$. Extrapolation of $\frac{\partial u}{\partial x}(1, t=1.6)$ yields the value -931.3647 with two significant digits.

Mesh adaptivity is required at lower values of the viscosity to resolve the exponential layer. In the one-dimensional case, this may be achieved by domain decomposition and local refinement close to the wall. For higher dimensions, the reader is referred for example to the paper by Sun et al. [12].

Another way to cope with this difficulty consists in using highorder methods like spectral elements or $h p$ methods.

\section{Conclusions}

The analytical solution of the linear advection-diffusion equation is obtained for Dirichlet boundary conditions and a smooth sine initial function. The closed form solution involves the Reynolds number as the governing parameter in exponential terms. The presence of these terms renders the problem stiff and ill-conditioned for small viscosity values. The comparison of analytical and FE solutions are presented for various values of the viscosity when the advection velocity is maintained constant. For moderate viscosity values $v<1 / 100 \pi$ both solutions are easily computed.

When viscosity goes to zero, another analytical approach is designed based on a perturbed wave equation. The solution is very close to the Fourier solution which would be obtained for periodic conditions.

For weak values of the viscosity, a downstream boundary layer builds up close to the boundary. This layer has an exponential characterization. Therefore the FE mesh needs local refinement to resolve for example the numerical evaluation of the slope next to the wall.

\section{Acknowledgment}

This research started when MOD was residing at the Université Paul Sabatier as visiting Professor. 


\section{References}

[1] Abramowitz M, Stegun IA. Handbook of mathematical functions with formulas graphs, and mathematical tables. 10th ed. New York: J Wiley; 1972.

[2] Basdevant C, Deville M, Haldenwang P, Lacroix JM, Ouazzani J, Peyret R, et al Spectral and finite difference solutions of the Burgers equation. Comput Fluids $1986 ; 14: 23-41$

[3] Bosen G. Method for the exact solution of a nonlinear diffusion-convection equation. Phys Rev Lett 1982;49(N25):1844-6.

[4] Cotta RM. Integral transforms in computational heat and fluid flow. Boca Raton (FL): CRC Press; 1993.

[5] Dehghan M. Weighted finite difference techniques for the one-dimensiona advection-diffusion equation. Appl Math Comput 2004;147:307-19.

[6] Donea J, Huerta A. Finite element methods for flow problems. Chichester (England): J Wiley; 2003.

[7] Gresho PM, Griffiths DF, Silvester DJ. Adaptive time-stepping for incompressible flow. Part I: Scalar advection-diffusion. SIAM J Sci Comput 2008;30:2018-54.

[8] Kumar A, Kumar Jaiswal D, Kumar N. Analytical solutions to one-dimensional advection-diffusion equation with variable coefficients in semi-infinite media. J Hydrol 2010;380:330-7.
9] Maple 17. Maplesoft, a division of Waterloo Maple Inc, Waterloo, Ontario.

[10] Pérez Guérrero JS, Pimentel LCG, Skaggs TH, van Genuchten MTh. Analytical solution of the advection-diffusion transport equation using a change-ofvariable and integral transform technique. Int $\mathrm{J}$ Heat Mass Trans 2009;52:3297-304.

[11] Pérez Guérrero JS, Pimentel LCG, Skaggs TH, Van Genuchten MTh. Analytical solution of the one-dimensional advection-dispersion solute transport equation subject to time boundary conditions. Chem Eng J 2013;221:487-91.

[12] Sun P, Chen L, Xu J. Numerical studies of adaptive finite element methods for two dimensional convection-dominated problems. J Sci Comput 2010;43:24-43.

[13] van Genuchten MTh, Leij FJ, Skaggs TH, Toride N, Bradford SA, Pontedeiro EM. Exact analytical solutions for contaminant transport in rivers. 1 . The equilibrium advection-diffusion equation. J Hydrol Hydromech 2013;61:146-60.

[14] Zoppou C, Knight JH. Analytical solution of a spatially variable coefficient advection-diffusion equation in up to three dimensions. Appl Math Model 1999;23:667-85. 